\title{
LA RUPTURE DES PLAQUES ET DES COQUES FISSURÉES
}

\author{
D. BERGEZ
}

\author{
Société Nationale des Pétroles d'Aquitaine \\ 26, avenue des Lilas, 64000 Pau, France.
}

\begin{abstract}
Résumé. - On donne, pour les plaques et les coques, la distribution des efforts et des moments tant en variation angulaire qu'en intensité. On décrit ensuite un critère permettant de prévoir la croissance des fissures.

Abstract. - In the case of plates and shells, we give the distribution of membrane forces, moments and shear forces as functions of polar coordinates. Path-independant integrals are given to determine the intensity factors. Then we study a fracture criterion for cracked bidimensional structures.
\end{abstract}

1. Introduction. - Il ne fait de doute pour personne que la présence d'une fissure dans une structure, si elle n'est pas souhaitable, n'a heureusement pas toujours des conséquences catastrophiques. En effet, on peut dans tous les cas tolérer les criques dont les dimensions sont compatibles avec le chargement pour une vitesse de croissance et des caractéristiques critiques du matériau données.

La connaissance du champ de contraintes au bout

la fissure est nécessaire pour prévoir l'évolution $\mathrm{du}$ défaut sous l'effet du chargement. Si, pour les problèmes plans, ce champ est bien connu, pour les coques la littérature n'en donne que des approximations. En effet, Folias [1-2] et Erdogan [3] ont étudié, à partir des équations de Marguerre [4], les cylindres fissurés sous des chargements particuliers. Ils trouvent que, bien que les efforts de membrane varient autour de la fissure comme les contraintes dans un problème plan, les moments ont, eux, des variations angulaires différentes. En outre, les efforts tranchants qu'ils mettent en évidence varient comme $r^{-3 / 2}$ et sont associés à des déformations de cisaillement nulles.

Pourtant, Knowles et Wang [5] avaient déjà trouvé, sur un cas particulier de flexion de plaque, des distributions angulaires de moments identiques à celles des efforts de membrane. Il est vrai qu'ils avaient abandonné la théorie de flexion des plaques généralement employée pour utiliser celle, plus complexe, de Reissner [6]. Cela signifie qu'il devait être possible de trouver en prenant une théorie des coques libérée de l'hypothèse de Kirchhoff, non seulement des distributions angulaires de moments identiques à celles des efforts de membrane, mais encore des efforts tranchants qui, associés à des déformations de cisaillement non nulles, donneront néanmoins une énergie de déformation sommable en fond de fissure.
Toutes ces études présentent des solutions globales, c'est-à-dire des solutions qui tiennent compte de la géométrie de toute la structure et de conditions aux limites données.

On peut montrer que, comme dans le cas plan, en se limitant aux termes singuliers, on résume dans un petit nombre de paramètres la géométrie de la coque et le chargement. Une telle étude, locale, permet de définir ces paramètres sans toutefois accéder à leur valeur.

Dans une seconde partie, il sera indiqué comment déterminer ces constantes au moyen d'intégrales indépendantes du chemin d'intégration. Le champ de contraintes, défini en variations angulaires et en intensité, sera alors parfaitement déterminé.

Mais nous ne saurons pas pour autant prévoir le chargement qui provoque l'instabilité de la fissure. Cela ne peut se faire qu'au moyen d'un critère de rupture et nous donnerons celui qui, pour les coques, nous semble être le plus adapté.

2. La caractérisation des fissures sur les coques. - Considérons (Fig. 1) une coque fissurée d'épaisseur $2 h$ soumise à un chargement quelconque. Nous rapporterons sa surface moyenne à un système de coordonnées curvilignes $x^{\alpha}(\alpha=1,2)$ de vecteurs de base $a_{\alpha}$. Le vecteur unitaire normal à la surface sera noté $a_{3}$ et sera tel que le trièdre $a_{1}, a_{2}, a_{3}$ soit direct. Les composantes deux fois covariantes des tenseurs métrique et de courbure seront $a_{\alpha \beta}$ et $b_{\alpha \beta}$.

Dans ces axes, le déplacement $u$ d'un point de la coque de coordonnées $\left(x^{\alpha}, x^{3}\right)$ peut être linéarisé en $x^{3}$ et prend alors la forme :

$$
\begin{aligned}
& u=u^{\alpha} a_{\alpha}+w a_{3}= \\
& \quad=\left[v^{\alpha}\left(x^{\beta}\right)+x^{3} \beta^{\alpha}\left(x^{\beta}\right)\right] a_{\alpha}+w\left(x^{\beta}\right) a_{3} .
\end{aligned}
$$




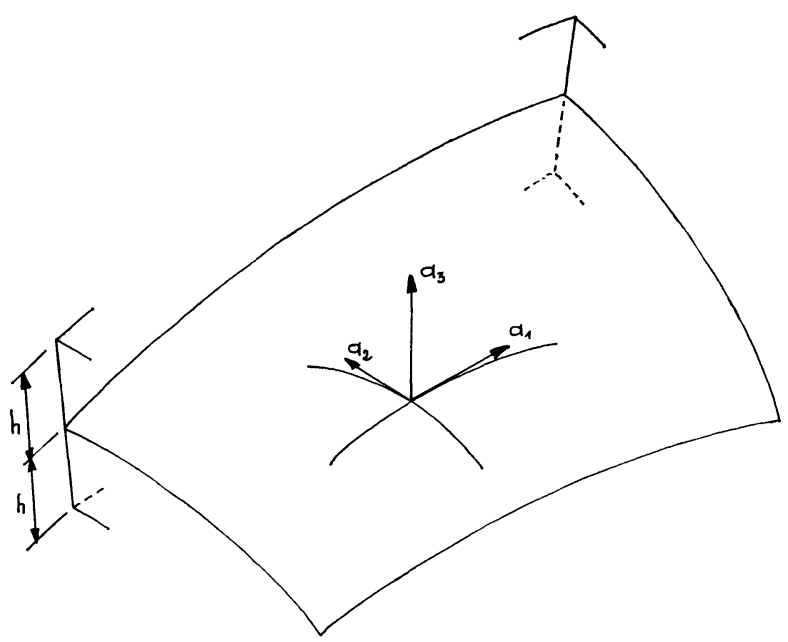

FIG. 1.

L'hypothèse de Kirchhoff qui veut que la normale matérielle avant déformation soit normale à la surface moyenne déformée revient à poser :

$$
\beta_{\alpha}=-w_{, \alpha}-b_{\alpha}^{\beta} v_{\beta} \text {. }
$$

Une théorie analogue à celle de Reissner pour les plaques (voir par exemple [10]) s'obtient en considérant $\beta_{\alpha}$ indépendant de $w$.

Ainsi, les déformations de membrane $\gamma_{\alpha \beta}$, les déformations liées aux variations de courbure $x_{\alpha \beta}$ et les rotations relatives moyennes de la normale matérielle $\gamma_{\alpha 3}$ sont données par les relations suivantes:

$$
\begin{aligned}
& \gamma_{\alpha \beta}=\left.v_{\alpha}\right|_{\beta}-b_{\alpha \beta} w \\
& \kappa_{\alpha \beta}=\left.\beta_{\alpha}\right|_{\beta} \\
& \gamma_{\alpha 3}=\beta_{\alpha}+w_{, \alpha}+b_{\alpha}^{\beta} v_{\beta} .
\end{aligned}
$$

Dans ces expressions, la virgule (,) et la barre (|) représentent respectivement les dérivations partielle et covariante.

$E$ et $v$ étant les constantes habituelles de l'élasticité, les équations de comportement donnant les efforts de membrane $N^{\alpha \beta}$, les moments $M^{\alpha \beta}$ et les efforts tranchants $Q^{\alpha}$ s'écrivent :

$$
\begin{aligned}
N^{\alpha \beta} & =\frac{E h}{1-v^{2}} A^{\beta \gamma \delta \mu}\left[\delta_{\gamma}^{\alpha} \gamma_{\delta \mu}+\frac{1}{12} I_{\gamma \mu}^{\alpha \nu}\left(b_{v}^{\rho} \gamma_{\delta \rho}+\kappa_{\delta v}\right)\right] \\
M^{\alpha \beta} & =\frac{E h^{3}}{12\left(1-v^{2}\right)} A^{\beta \gamma \delta \mu}\left[\delta_{\gamma}^{\alpha} \kappa_{\delta \mu}+I_{\delta \mu}^{\alpha \nu} \gamma_{\delta v}\right] \\
Q^{\alpha} & =\frac{5 E h}{12(1+v)} a^{\beta \gamma}\left[\delta_{\beta}^{\alpha} \delta_{\gamma}^{\mu}+\frac{h^{2}}{28} I_{\beta \nu}^{\alpha \mu} b_{\gamma}^{\nu}\right] \gamma_{\mu 3} .
\end{aligned}
$$

Lorsque les lignes de coordonnées choisies sont des lignes de courbure, et appelant $R_{1}$ et $R_{2}$ les rayons de courbure principaux, $A^{\alpha \beta \gamma \delta}$ et $I_{\gamma \delta}^{\alpha \beta}$ sont définis de la façon suivante :

$$
\begin{gathered}
A^{\alpha \beta \gamma \delta}=v a^{\alpha \beta} a^{\gamma \delta}+\frac{1-v}{2}\left[a^{\alpha \delta} a^{\beta \gamma}+a^{\alpha \gamma} a^{\beta \nu}\right] \\
I_{\gamma \delta}^{\alpha \beta}=\left\{\begin{array}{l}
\frac{1}{R_{2}}-\frac{1}{R_{1}} \text { pour } \alpha=\beta=\gamma=\delta=1 \\
\frac{1}{R_{1}}-\frac{1}{R_{2}} \text { pour } \alpha=\beta=\gamma=\delta=2 \\
0 \text { dans tous les autres cas }
\end{array}\right.
\end{gathered}
$$

$p$ désignant une pression appliquée sur une des faces de la coque, les équations de l'équilibre s'écrivent :

$$
\begin{aligned}
& \left.N^{\beta \alpha}\right|_{\beta}-b_{\gamma}^{\alpha} Q^{\gamma}=0 \\
& \left.M^{\beta \alpha}\right|_{\beta}-Q^{\alpha}=0 \\
& \left.Q^{\alpha}\right|_{\alpha}+b_{\alpha \gamma} N^{\gamma \alpha}+p=0 .
\end{aligned}
$$

En procédant comme dans [7] et [8], on porte les relations de comportement exprimées en déplacements dans les équations de l'équilibre et on obtient les cinq équations différentielles qui régissent le problème. Pour une étude locale, il suffit de garder, dans ces équations, les termes présentant la singularité la plus forte, c'est-à-dire ceux dont le degré de dérivation partielle est le plus élevé. On constate alors la disparition des termes contenant la courbure et ceux contenant la métrique. On peut, dans ces conditions, utiliser des coordonnées cartésiennes liées au fond de la fissure et aligner les indices. On obtient :

$$
\begin{aligned}
(1-v) v_{\alpha, \beta \beta}+(1+v) v_{\beta, \alpha \beta} & =0 \\
(1-v) \beta_{\alpha, \beta \beta}+(1+v) \beta_{\beta, \alpha \beta} & =0 \\
w_{, \alpha \alpha} & =0 .
\end{aligned}
$$

Pour intégrer ce système, on passe en coordonnées polaires locales et on recherche les solutions sous la forme $r^{\alpha} f(\theta)$. Il est bon de remarquer que la présence de la fissure interdit aux fonctions $f(\theta)$ d'être $2 \pi$ périodiques car les points $\theta=\pi$ et $\theta=-\pi$ bien que géométriquement identiques sont matériellement différents. La première périodicité acceptable est donc $4 \pi$. La résolution du système différentiel montre que, pour qu'il en soit ainsi, $\alpha$ doit être de la forme $n / 2$, $n$ étant un entier. Il est, de plus, aisé de constater que pour $n \leqslant 0$ la densité d'énergie de déformation n'est pas sommable autour de la fissure et que pour $n \geqslant 2$ il n'y a pas de singularité. Nous retenons donc $n=1$, soit $\alpha=\frac{1}{2}$, et obtenons ainsi le même résultat que pour le plan. Les trois composantes du déplacement d'un point sont alors les suivantes : 


$$
\begin{gathered}
\begin{aligned}
\frac{2 E}{1+v} u_{1}=\left(K_{\mathrm{s}}+\frac{2 x^{3}}{h} K_{\mathrm{s}}^{*}\right)\left(\frac{r}{2 \pi}\right)^{1 / 2}\left[(2 \kappa-1) \operatorname{Cos} \frac{\theta}{2}-\operatorname{Cos} \frac{3 \theta}{2}\right]+ & +\left(K_{\mathrm{a}}+\frac{2 x^{3}}{h} K_{\mathrm{a}}^{*}\right)\left(\frac{r}{2 \pi}\right)^{1 / 2}\left[(2 \kappa+3) \operatorname{Sin} \frac{\theta}{2}+\operatorname{Sin} \frac{3 \theta}{2}\right] \\
\frac{2 E}{1+v} u_{2}=\left(K_{\mathrm{s}}+\frac{2 x^{3}}{h} K_{\mathrm{s}}^{*}\right)\left(\frac{r}{2 \pi}\right)^{1 / 2}\left[(2 \kappa+1) \operatorname{Sin} \frac{\theta}{2}-\operatorname{Sin} \frac{3 \theta}{2}\right]+ & +\left(K_{\mathrm{a}}+\frac{2 x^{3}}{h} K_{\mathrm{a}}^{*}\right)\left(\frac{r}{2 \pi}\right)^{1 / 2}\left[(3-2 \kappa) \operatorname{Cos} \frac{\theta}{2}-\operatorname{Cos} \frac{3 \theta}{2}\right] \\
\frac{5 E}{16(1+v)} w= & K_{\mathrm{a}}^{* *}\left(\frac{r}{2 \pi}\right)^{1 / 2} \operatorname{Sin} \frac{\theta}{2} \\
\kappa & =\frac{3-v}{1+v} .
\end{aligned}
\end{gathered}
$$

Dans ces expressions, $K_{\mathrm{s}}$ et $K_{\mathrm{a}}$ jouent le même rôle que $K_{\mathrm{I}}$ et $K_{\mathrm{II}}$ dans les problèmes plans. $K_{\mathrm{s}}^{*}$ et $K_{\mathrm{a}}^{*}$ sont les facteurs d'intensité des moments dans les cas symétrique et antisymétrique, tandis que $K_{\mathrm{a}}^{* *}$ correspond au mode III. Notons que sur les coques on ne peut séparer que deux modes: symétrique $\left(K_{\mathrm{s}}, K_{\mathrm{s}}^{*}\right)$ et antisymétrique $\left(K_{\mathrm{a}}, K_{\mathrm{a}}^{*}, K_{\mathrm{a}}^{* *}\right)$.

3. Le calcul des facteurs d'intensité des contraintes. - Lorsqu'il s'agit de problèmes plans, il est à l'heure actuelle très courant, tant du point de vue du calcul que de celui de l'expérience, d'utiliser l'intégrale de Rice $J_{x}$ [9] (Fig. 2) :

$$
J_{x}=\int_{\Gamma}\left[W \mathrm{~d} x^{2}-\sigma_{\alpha \beta} n_{\alpha} u_{\beta, 1} \mathrm{~d} s\right] .
$$

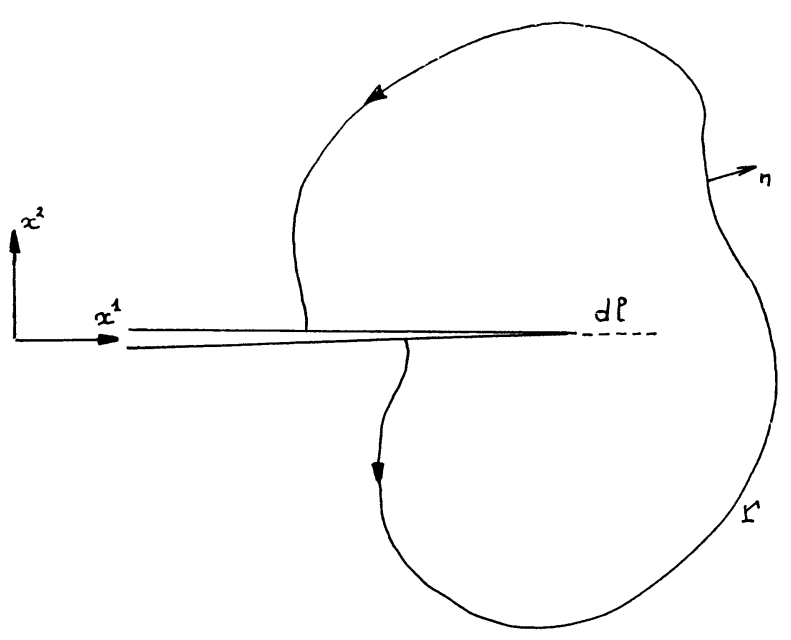

FIG. 2.

Cette intégrale, fonction de la densité d'énergie de déformation $W$, possède deux propriétés : elle est indépendante du chemin d'intégration $\Gamma$ et est égale au taux de croissance de l'énergie potentielle $P$ par rapport à la longueur $l$ de la fissure $(-\mathrm{d} P / \mathrm{d} l)$ lorsque celle-ci croît dans son prolongement.
De plus, en contrainte plane, elle est reliée aux facteurs d'intensité des contraintes $K_{\mathrm{I}}$ et $K_{\mathrm{II}}$ par :

$$
J_{x}=\frac{K_{\mathrm{I}}^{2}+K_{\mathrm{II}}^{2}}{E} .
$$

Par conséquent, dans le cas où un seul des modes intervient, la connaissance de $J_{x}$ donne accès au $K$. Par contre, pour des modes combinés, nous ne pouvons pas séparer $K_{\mathrm{I}}$ de $K_{\mathrm{II}}$. Pour y arriver, nous allons étendre la notion de $J_{x}$ au cas où il y a changement brutal de direction (Fig. 3).

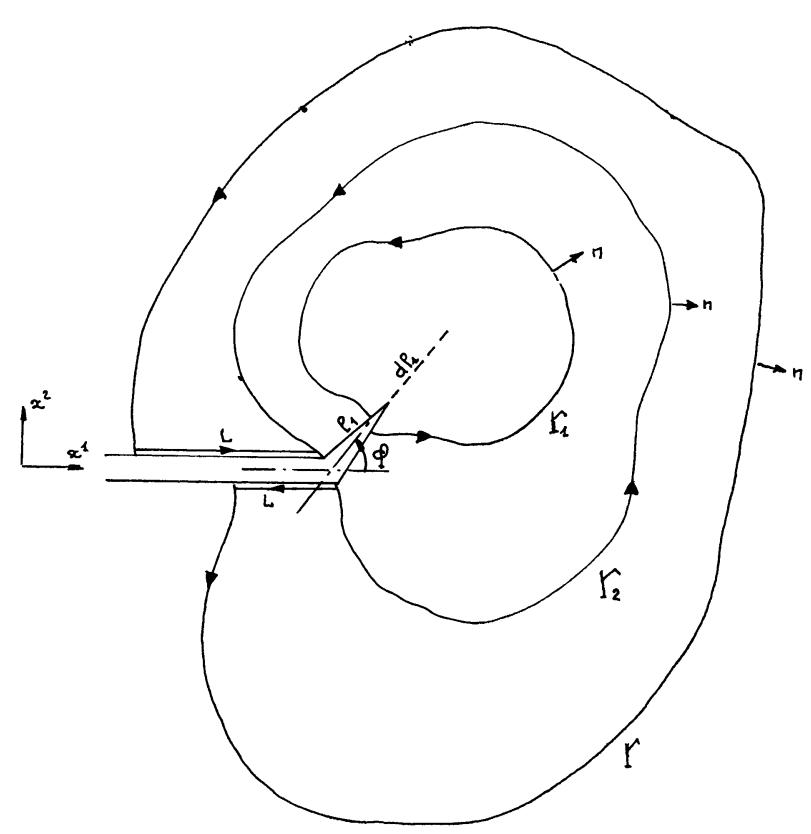

FIG. 3.

Considérons une fissure de longueur $l_{1}$ faisant un angle $\varphi$ avec $O x^{1}$. Un simple changement d'axes montre que l'on a : 


$$
\begin{aligned}
-\frac{\mathrm{d} P}{\mathrm{~d} l_{1}}= & \operatorname{Cos} \varphi\left\{\int_{\Gamma_{1}}\left[W \mathrm{~d} x^{2}-\sigma_{\alpha \beta} n_{\alpha} u_{\beta, 1} \mathrm{~d} s\right]\right\}+ \\
& +\operatorname{Sin} \varphi\left\{\int_{\Gamma_{1}}\left[-W \mathrm{~d} x^{1}-\sigma_{\alpha \beta} n_{\alpha} u_{\beta, 2} \mathrm{~d} s\right]\right\}
\end{aligned}
$$

ou, avec des notations évidentes :

$$
-\frac{\mathrm{d} P}{\mathrm{~d} l_{1}}=J_{x} \operatorname{Cos} \varphi+J_{y}^{*} \operatorname{Sin} \varphi .
$$

Tant que ses extrémités sont sur la branche $l_{1}$, l'expression ci-dessus ne dépend pas de $\Gamma_{1}$ alors que $J_{x}$ et $J_{y}^{*}$ dépendent du contour.

On peut donc définir $-\mathrm{d} P / \mathrm{d} l_{1}$ sur un contour $\Gamma_{2}$ dont les extrémités sont sur le changement de direction de la fissure.

Si maintenant on veut rattacher $-\mathrm{d} P / \mathrm{d} l_{1}$ défini sur $\Gamma_{2}$ à des intégrales définies sur $\Gamma$ dont les extrémités sont sur la branche de la fissure portée par $O x^{1}$, on peut montrer que l'on a :

$$
\begin{aligned}
-\frac{\mathrm{d} P}{\mathrm{~d} l_{1}}= & \operatorname{Cos} \varphi\left\{\int_{\Gamma}\left[W \mathrm{~d} x^{2}-\sigma_{\alpha \beta} n_{\alpha} u_{\beta, 1} \mathrm{~d} s\right]\right\}+ \\
& +\operatorname{Sin} \varphi\left\{\int_{\Gamma}\left[-W \mathrm{~d} x^{1}-\sigma_{\alpha \beta} n_{\alpha} u_{\beta, 2} \mathrm{~d} s\right]\right. \\
& \left.+\int_{L}\left[-W \mathrm{~d} x^{1}\right]\right\} \\
= & \operatorname{Cos} \varphi J_{x}+\operatorname{Sin} \varphi J_{y} .
\end{aligned}
$$

Par contre, maintenant, $J_{x}$ et $J_{y}$ sont indépendants de $\Gamma$.

En outre, comme $-\mathrm{d} P / \mathrm{d} l_{1}$ ne dépend pas de $l_{1}$, nous pouvons dire que nous disposons de deux intégrales indépendantes du chemin d'intégration et reliées au taux de décroissance de l'énergie potentielle : $J_{x}$ lorsque la fissure ne change pas de direction et, en faisant tendre $l_{1}$ vers zéro, $J_{y}$ lorsque la propagation est perpendiculaire à son plan initial. Pour une croissance dans la direction $\varphi$ on a :

$$
-\frac{\mathrm{d} P}{\mathrm{~d} l}(\varphi)=J_{x} \operatorname{Cos} \varphi+J_{y} \operatorname{Sin} \varphi .
$$

Restant toujours en contrainte plane, $J_{y}$ s'exprime en fonction de $K_{\mathrm{I}}$ et $K_{\mathrm{II}} \mathrm{par}$ :

$$
J_{y}=-2 \frac{K_{\mathrm{I}} K_{\mathrm{II}}}{E} .
$$

La connaissance de $J_{x}$ et $J_{y}$ permet donc de déterminer $K_{\mathrm{I}}$ et $K_{\mathrm{II}}$.

Nous pouvons étendre ces notions aux coques. En effet, supposant que la fissure est portée par $x^{1}$, nous appellerons $l^{*}$ non pas la longueur de la fissure mais l'abscisse de son extrémité et $\bar{\Sigma}$ l'énergie de déformation par unité d'aire de la surface moyenne. Alors $J_{x}$ et $J_{y}$, définis par les relations ci-dessous, sont indépendantes du chemin d'intégration et reliées, comme dans le plan à la décroissance de l'énergie potentielle.

$$
\begin{gathered}
J_{x}=-\frac{\mathrm{d} P}{\mathrm{~d} l^{*}}\left(x^{2}=0\right)= \\
=\int_{\Gamma}\left[\bar{\Sigma} \mathrm{d} x^{2}-\left(\left.N^{\alpha v} v_{v}\right|_{1}+\left.M^{\alpha v} \beta_{v}\right|_{1}+Q^{\alpha} w_{, 1}\right) n_{\alpha} \mathrm{d} s\right] \\
J_{y}=-\frac{\mathrm{d} P}{\mathrm{~d} l^{*}}\left(x^{1}=l^{*}\right)= \\
=\int_{\Gamma}\left[-\bar{\Sigma} \mathrm{d} x^{1}-\left(\left.N^{\alpha v} v_{v}\right|_{2}+\left.M^{\alpha v} \beta_{v}\right|_{2}+Q^{\alpha} w_{, 2}\right) n_{\alpha} \mathrm{d} s\right]+ \\
+\int_{L}\left[-\bar{\Sigma} \mathrm{d} x^{1}\right] .
\end{gathered}
$$

Dans ce cas, on dispose toujours, pour calculer les facteurs $K$, de deux équations alors que le nombre des inconnues est passé à 5 . Nous avons là, en fait, des intégrales qui, en plus d'une propriété mathématique d'indépendance à l'égard de $\Gamma$, ont un sens physique de décroissance d'énergie. Mais, pour calculer les facteurs $K$, point n'est besoin d'intégrales ayant un sens physique ; la propriété d'indépendance suffit.

On peut, à titre d'exemple, donner la façon dont on résout entièrement le problème des plaques. Il suffit de remarquer que, dans $\bar{\Sigma}$, les termes de membrane, de flexion et d'efforts tranchants s'ajoutent sans couplage. Par conséquent, toutes les intégrales ci-dessous sont indépendantes de $\Gamma$ :

$$
\begin{array}{ll}
\int_{\Gamma}\left[\frac{1}{2} N^{\alpha \beta} \gamma_{\alpha \beta} \mathrm{d} x^{2}-\left.N^{\alpha \beta} v_{\beta}\right|_{1} n_{\alpha} \mathrm{d} s\right] & =\frac{\left(K_{\mathrm{s}}\right)^{2}+\left(K_{\mathrm{a}}\right)^{2}}{E} \\
\int_{\Gamma}\left[-\frac{1}{2} N^{\alpha \beta} \gamma_{\alpha \beta} \mathrm{d} x^{1}-\left.N^{\alpha \beta} v_{\beta}\right|_{2} n_{\alpha} \mathrm{d} s\right]+\int_{L}-\frac{1}{2} N^{\alpha \beta} \gamma_{\alpha \beta} \mathrm{d} x^{1} & =\frac{-2 K_{\mathrm{s}} K_{\mathrm{a}}}{E} \\
\int_{\Gamma}\left[\frac{1}{2} M^{\alpha \beta} \kappa_{\alpha \beta} \mathrm{d} x^{2}-\left.M_{\alpha \beta} \beta_{\beta}\right|_{1} n_{\alpha} \mathrm{d} s\right] & =\frac{\left(K_{\mathrm{s}}^{*}\right)^{2}+\left(K_{\mathrm{a}}^{*}\right)^{2}}{3 E} \\
\int_{\Gamma}\left[-\frac{1}{2} M^{\alpha \beta} \kappa_{\alpha \beta} \mathrm{d} x^{1}-\left.M^{\alpha \beta} \beta_{\beta}\right|_{2} n_{\alpha} \mathrm{d} s\right]+\int_{L}-\frac{1}{2} M^{\alpha \beta} \kappa_{\alpha \beta} \mathrm{d} x^{1}=\frac{-2 K_{\mathrm{s}}^{*} K_{\mathrm{a}}^{*}}{3 E}
\end{array}
$$




$$
\begin{array}{ll}
\int_{\Gamma}\left[\frac{1}{2} Q^{\alpha} \gamma_{\alpha 3} \mathrm{~d} x^{2}-Q^{\alpha} w_{, 1} n_{\alpha} \mathrm{d} s\right] & =\frac{8}{15 E}(1+v)\left(K_{\mathrm{a}}^{* *}\right)^{2} \\
\int_{\Gamma}\left[-\frac{1}{2} Q^{\alpha} \gamma_{\alpha 3} \mathrm{~d} x^{1}-Q^{\alpha} w_{, 2} n_{\alpha} \mathrm{d} s\right]+\int_{L}-\frac{1}{2} Q^{\alpha} \gamma_{\alpha 3} \mathrm{~d} x^{1} & =0 .
\end{array}
$$

Les cinq premières équations permettent de déterminer les cinq facteurs d'intensité des contraintes. Notons cependant que la question mérite d'être approfondie.

4. Les critères de rupture. - Une fois déterminé le champ de contraintes au voisinage du fond de la fissure, il faut savoir comment utiliser les résultats précédents pour, d'une part, prévoir la direction de la croissance de la crique et, d'autre part, le chargement critique. Ceci se fait à l'aide d'un critère de rupture.

On trouve, dans la littérature, plusieurs critères permettant de répondre aux deux questions posées dans le cas de problème plan. Une étude critique montre que, dans le plan, le critère proposé par Mandel J. (Communication privée) donne les meilleurs résultats.

Soient $\sigma_{\theta \theta}$ et $\tau_{r \theta}$ les composantes polaires des efforts exercés sur une facette passant par le fond de la fissure et faisant un angle $\theta$ avec elle. Considérons alors (Fig. 4) le repère $\langle x, y\rangle$ défini par :

$$
\begin{aligned}
& x=4(2 \pi r)^{1 / 2} \sigma_{\theta \theta} \\
& y=4(2 \pi r)^{1 / 2} \tau_{r \theta} .
\end{aligned}
$$

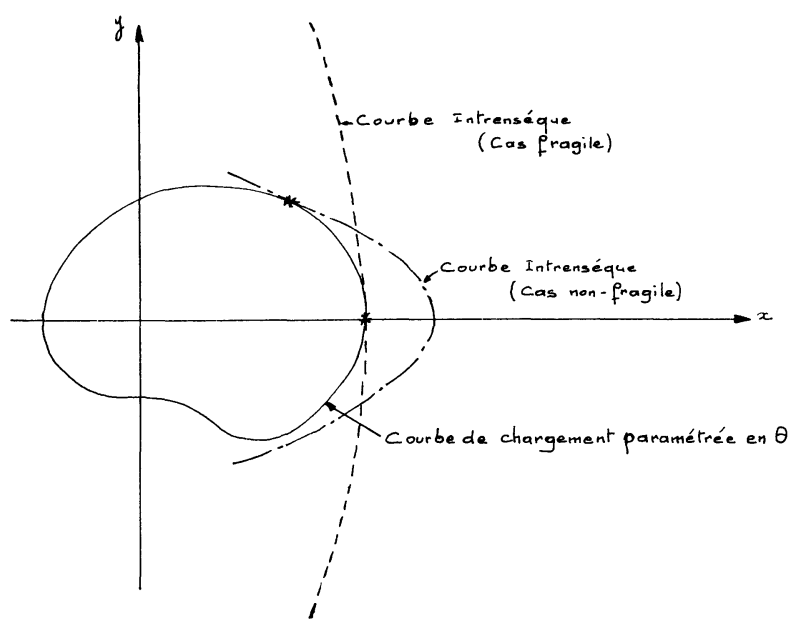

FIG. 4.

Dans ce plan, le chargement de la fissure est représenté par une courbe paramétrée en $\theta$ et le critère proposé par Mandel s'énonce ainsi :

La fissure commence à croître lorsque la courbe représentative de l'état des contraintes dans le plan $\langle x, y\rangle$ sera tangente à une certaine courbe carac- térisant la résistance moléculaire du matériau. $\mathbf{L a}$ valeur de $\theta$ au point de tangence donnera la direction de la croissance.

S'il est relativement facile d'obtenir la courbe de chargement, on ne saurait en dire autant de la courbe intrinsèque de résistance moléculaire. Mais cette définition générale du critère englobe un cas particulier important qu'il convient de noter.

Mandel démontre en effet que les tangentes à la courbe de chargement sur $O x$ sont parallèles à $O y$ et que la courbe intrinsèque est symétrique par rapport à $O x$. En outre, il introduit l'hypothèse que cette dernière est suffisamment ouverte pour que le contact avec la courbe de chargement ait lieu sur $O x$. Le critère devient :

$$
\tau_{r \theta}=0, \quad \sigma_{\theta \theta}=\sigma_{\text {critique }} .
$$

On retrouve ainsi le critère de la plus grande contrainte normale mais on procure une définition de la fragilité qui s'appuie sur le rayon de courbure de la courbe intrinsèque pour $y=0$.

Nous avons étendu ce critère aux coques. Sans entrer dans les détails, disons qu'il existe trois cas distincts :

- Lorsque les efforts tranchants $\left(K_{\mathrm{a}}^{* *}\right)$ peuvent être négligés, on se ramène à un problème plan en posant :

$$
\begin{aligned}
& K_{\mathrm{I}}=K_{\mathrm{s}}+\frac{2 x^{3}}{h} K_{\mathrm{s}}^{*} \\
& K_{\mathrm{II}}=K_{\mathrm{a}}+\frac{2 x^{3}}{h} K_{\mathrm{a}}^{*} .
\end{aligned}
$$

Lorsque les efforts tranchants sont prépondérants, comme par exemple dans un mode III pur, la rupture aura lieu pour une valeur critique de $K_{\mathrm{a}}^{* *}$. Cette dernière n'est d'ailleurs pas nécessairement $K_{\mathrm{c}}$.

- Dans tous les cas intermédiaires, il est nécessaire de connaitre la courbe intrinsèque.

Pour des coques, en plus de la direction de la croissance, il faut déterminer la valeur de $x^{3}$ pour laquelle elle aura lieu. Dans le plan $\langle x, y\rangle$, le chargement est représenté non plus par une courbe, comme dans les problèmes plans, mais par une famille de courbes à un paramètre qui est $x^{3}$.

5. Conclusion. - L'étude présente, dans laquelle nous avons défini les facteurs d'intensité des contraintes dans les coques ( $(2)$, indiqué un moyen de les cal- 
culer (§ 3) et proposé le critère de rupture correspondant $(\S 4)$, avait pour but de dégager des bases sûres pour la détermination des risques de rupture d'une coque. Nous sommes bien conscients du fait que ce travail doit être prolongé par des applications pratiques.

\section{Bibliographie}

[1] Fol:4S, E. S., Int. J. Fracture Mechanics 1 (1965) 104.

[2] Folias, E. S., Int. J. Fracture Mechanics 3 (1967) 1.

[3] Erdogan, F. et Ratwani, M., Int. J. Fracture Mechanics 8 (1972) 87.

[4] Marguerre, K., Proc. 5th Int. Cong. Applied Mechanics (1938) 93.

[5] KNowles, J. K. et WANG, N. M., Journ. Math. Phys. 39 (1960) 223.
[6] Reissner, E., J. Appl. Mechanics 12 (1945) A 69.

[7] Bergez, D. et Radenkovic, D., C. R. Hebd. Sean. Acad. Sci. 275 (1972) A 221.

[8] Bergez, D. et Radenkovic, D., Proc. 2nd Int. Conf. Pressure Vessel Technology, p. 1089, 1973.

[9] Rice, J., Fracture 2 (1968) 191.

[10] Naghdi, P. M., Prog. Solid. Mech. 4 (1963). 